\title{
ARTICLE OPEN Multiple low-temperature skyrmionic states in a bulk chiral magnet
}

\author{
Lars J. Bannenberg ${ }^{1}$, Heribert Wilhelm (D) $^{2,7}$, Robert Cubitt $\mathbb{D}^{3}$, Ankit Labh ${ }^{1}{ }^{1}$, Marcus P. Schmidt ${ }^{4}$, Eddy Lelièvre-Berna iD $^{3}$, \\ Catherine Pappas ${ }^{1}$, Maxim Mostovoy ${ }^{5}$ and Andrey O. Leonov ${ }^{6}$
}

Magnetic skyrmions are topologically protected nanoscale spin textures with particle-like properties. In bulk cubic helimagnets, they appear under applied magnetic fields and condense spontaneously into a lattice in a narrow region of the phase diagram just below the magnetic ordering temperature, the so-called A-phase. Theory, however, predicts skyrmions to be locally stable in a wide range of magnetic fields and temperatures. Our neutron diffraction measurements reveal the formation of skyrmion states in large areas of the magnetic phase diagram, from the lowest temperatures up to the A-phase. We show that nascent and disappearing spiral states near critical lines catalyze topological charge changing processes, leading to the formation and destruction of skyrmionic states at low temperatures, which are thermodynamically stable or metastable depending on the orientation and strength of the magnetic field. Skyrmions are surprisingly resilient to high magnetic fields: the memory of skyrmion lattice states persists in the field polarized state, even when the skyrmion lattice signal has disappeared. These findings highlight the paramount role of magnetic anisotropies in stabilizing skyrmionic states and open up new routes for manipulating these quasi-particles towards energy-efficient spintronics applications.

npj Quantum Materials (2019)4:11 ; https://doi.org/10.1038/s41535-019-0150-7

\section{INTRODUCTION}

Skyrmions were introduced into science by T.H.R. Skyrme who has put forward a non-linear meson model in which baryons, such as protons and neutrons, emerge as topological solitons. ${ }^{1}$ Importantly, he identified the baryon number with the topological charge of configurations of the meson field. The Skyrme model was applied to describe physical properties of baryons and atomic nuclei, ${ }^{2}$ as well as creation and annihilation of baryons assisted by monopoles. ${ }^{3}$ Related topological objects have been discovered in many branches of science, including condensed matter physics. ${ }^{4-7}$ The recent observation of magnetic skyrmions, ${ }^{8,9}$ which are topologically protected nanoscale spin textures, initiated a new rapidly expanding field of research, called skyrmionics. This field is driven by the exploration of new skyrmion materials and novel transport phenomena-quantum manisfestations of the nontrivial skyrmion topology-as well as by the potential of magnetic skyrmions to revolutionize information technologies. The use of skyrmions as information bits holds promise of fast, energyefficient and high-density magnetic memory devices. ${ }^{10-14}$

In bulk cubic helimagnets, where magnetic skyrmions were first spotted, ${ }^{8}$ skyrmion lattices (SkLs) appear spontaneously in a small pocket of the temperature-magnetic field phase diagram, the socalled A-phase, just below the transition temperature $T_{\mathrm{C}}$. Their stability is attributed to the competition between the ferromagnetic exchange, the Dzyaloshinskii-Moriya (DM) and Zeeman interactions in combination with additional small terms ${ }^{15}$ such as thermal fluctuations, ${ }^{8,16}$ dipolar interactions and the softening of the magnetization modulus. ${ }^{17,18}$ As the free energy imbalance between the skyrmion lattice phase and its main competitor, the conical state, is small, the boundaries of the A-phase can be changed substantially by applying pressure, ${ }^{19}$ electric fields, ${ }^{13,20-22}$ chemical doping, ${ }^{23}$ or uniaxial strains. ${ }^{24,25}$ On the other hand, theoretical models that are based on the Dzyaloshinskii approach $^{26,27}$ and include magnetic stiffness or anisotropy, predict SkLs to occur in bulk cubic helimagnets over a wide range of magnetic fields and temperatures below $T_{\mathrm{C}}{ }^{15,28-32}$

Experimental efforts to find anisotropy-stabilized low-temperature SkLs in bulk cubic helimagnets have been largely overshadowed by the search for SkLs and isolated skyrmions in thin films, ${ }^{9,14,33}$ where the main stabilization mechanism is the chiral surface twists. ${ }^{34-36}$ Nevertheless, the importance of anisotropy in stabilizing states is underscored by the recent discovery of the tilted spiral state in $\mathrm{Cu}_{2} \mathrm{OSeO}_{3}{ }^{37,38}$ resulting from the competition between the anisotropic exchange and the cubic anisotropy, which is very strong in that material due to the quantum nature of spins of its magnetic building blocks. ${ }^{37,39}$ Furthermore, similar mechanisms possibly stabilize the low-temperature skyrmion lattice reported in the same material. ${ }^{38}$

In the following, we present an experimental and theoretical study of skyrmionic states in the chiral magnetic insulator $\mathrm{Cu}_{2} \mathrm{OSeO}_{3}$ and discuss the stabilization and nucleation mechanisms thereof. Small angle neutron scattering (SANS) measurements were performed by carefully applying the magnetic field along the three major crystallographic directions, either after

\footnotetext{
${ }^{1}$ Faculty of Applied Sciences, Delft University of Technology, Mekelweg 15, 2629 JB Delft, The Netherlands; ${ }^{2}$ Diamond Light Source Ltd., OX11 0DE Didcot, UK; ${ }^{3}$ Institut LaueLangevin, 71 Avenue des Martyrs, CS 20156, 38042 Grenoble, France; ${ }^{4}$ Max Planck Institute for Chemical Physics of Solids, 01187 Dresden, Germany; ${ }^{5}$ Zernike Institute for Advanced Materials, University of Groningen, 9700 AB Groningen, The Netherlands and ${ }^{6}$ Chiral Research Center, Hiroshima University, Higashi Hiroshima, Hiroshima 739-8526, Japan

Correspondence: Catherine Pappas (c.pappas@tudelft.nl)

${ }^{7}$ Present address: Helmholtz-Institut Ulm, Helmholtzstraße 11, 89081 Ulm, Germany
}

Received: 4 December 2018 Accepted: 12 March 2019

Published online: 01 April 2019 
zero-field cooling (ZFC) or after fast-field cooling (FFC) through the A-phase. ${ }^{40-44}$ In this way, extremely robust low-temperature skyrmionic states can be induced for all field directions and over

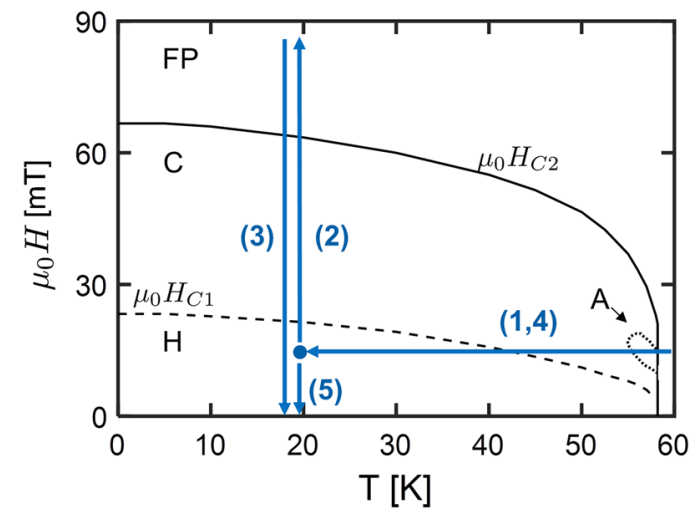

Fig. 1 Schematic representation of the fast field cooled (FFC) experimental procedure. The sample was (1) first field cooled under a magnetic field of $\mu_{0} \mathrm{H}=14 \mathrm{mT}$ to the temperature of interest with an average cooling rate of $10 \mathrm{~K} / \mathrm{s}$. Subsequently, (2) measurements were performed by stepwise increasing the magnetic field to $\mu_{0} H=$ $90 \mathrm{mT}$, well above the $\mu_{0} H_{\mathrm{C} 2}$ line. Next, (3) measurements were performed by stepwise decreasing the magnetic field to $\mu_{0} H=0 \mathrm{mT}$. In an additional step, the sample was brought to $T \gg T_{C}$, (4) field cooled again under $\mu_{0} \mathrm{H}=14 \mathrm{mT}$ and (5) measurements were performed by stepwise decreasing the field to $\mu_{0} H=0 \mathrm{mT}$. This figure also indicates the boundaries of the helimagnetic phases generic to cubic helimagnets: the Helical phase $(\mathrm{H})$ for $\mu_{0} H<\mu_{0} H_{\mathrm{C} 1}$, the Conical phase (C) for $\mu_{0} H_{C 1}<\mu_{0} H<\mu_{0} H_{C 2}$, the field polarized state (FP) for $\mu_{0} H>\mu_{0} H_{C 2}$ and the A-phase (A) close to $T_{C}$ large areas of the phase diagram, stabilized by competing cubic and exchange anisotropies. In particular, when the magnetic field is applied along the cubic axes, triangular arrays of skyrmions are energetically more favorable than both the tilted spiral and conical states near the borderline between modulated and uniform magnetic phases. Our theoretical approach shows that the spatial orientation of the skyrmion lattice is, in low magnetic fields, determined by magnetic anisotropies and not necessarily orthogonal to the applied field. Although emerging and disappearing spiral states near critical magnetic fields act as catalyzers for topological charge changing processes, skyrmions are surprisingly resilient to high magnetic fields: the memory of skyrmionic states persists, possibly in the form of isolated skyrmions or torons, in the field polarized state, even when the skyrmion lattice signal has disappeared. Our findings highlight the paramount role of the strong interplay between the competing magnetic anisotropies resulting from the quantum nature of spin in $\mathrm{Cu}_{2} \mathrm{OSeO}_{3}{ }^{37,39}$ in stabilizing skyrmionic states, which opens new routes for the manipulation of skyrmions in spintronic devices.

\section{RESULTS}

Figure 1 schematically illustrates our measurement protocols as well as the boundaries of the phases generic to cubic helimagnets. For the FFC measurements the sample was cooled to the temperature of interest with an average cooling rate of $10 \mathrm{~K} / \mathrm{s}$ and under a magnetic field of $\mu_{0} \mathrm{H}=14 \mathrm{mT}$. The ZFC measurements were performed by cooling the sample from $T \gg T_{C}$ to the temperature of interest at zero magnetic field following the protocols schematically illustrated in Fig. S6 of the Supplementary Information. The measurements were performed with the magnetic field either perpendicular or parallel to the incident

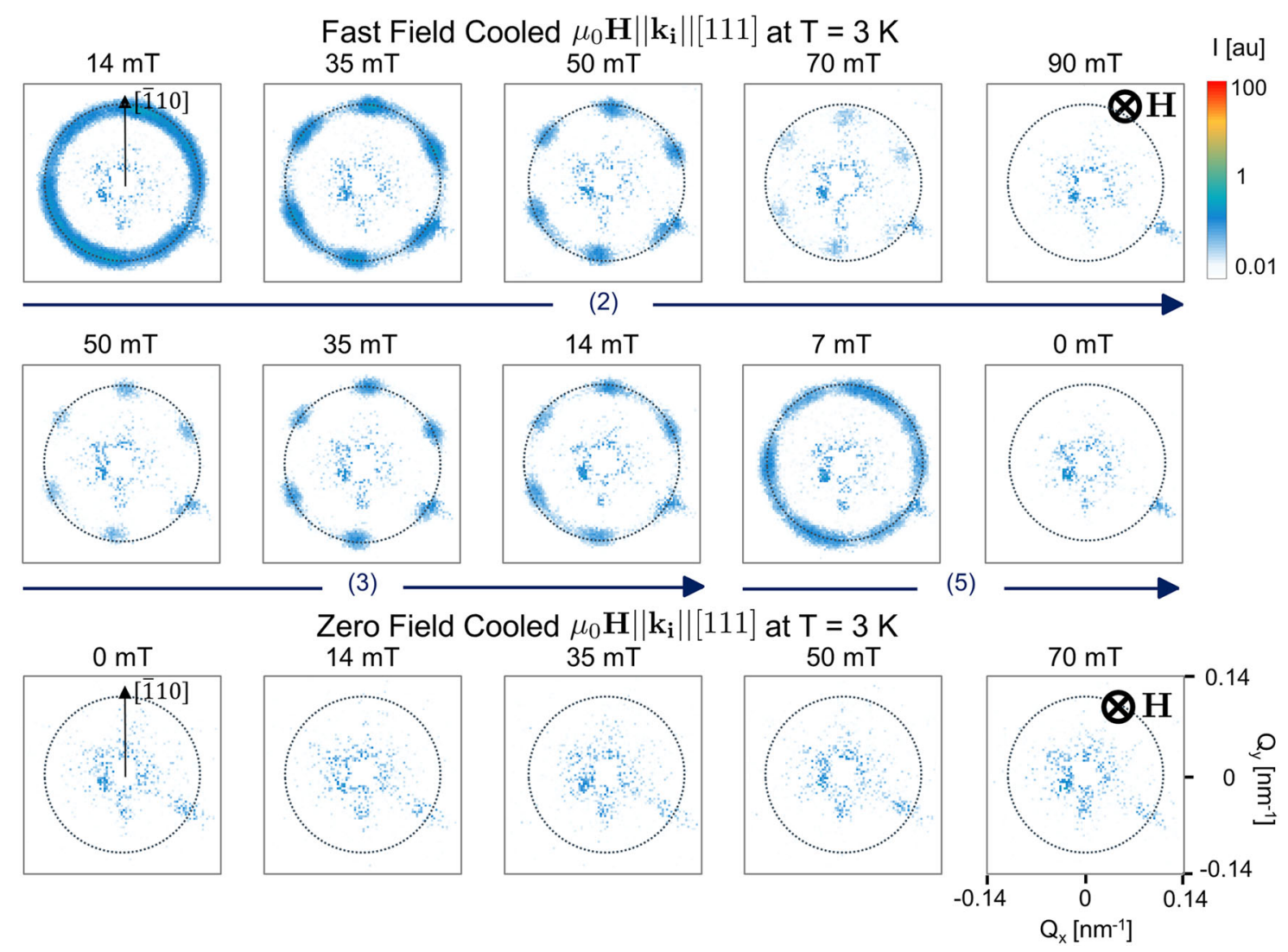

Fig. 2 SANS patterns recorded at $T=3 \mathrm{~K}$ and with the magnetic field applied along the [111] crystallographic direction. The magnetic field was applied parallel to the incoming neutron beam ( $\left.\mathbf{H}|| \mathbf{k}_{\mathbf{i}}\right)$ following the fast field cooled (FFC) and zero field cooled protocols. The arrows and numbers below the SANS patterns indicate the step of the FFC protocol, as defined in Fig. 1, for which the measurements were performed 


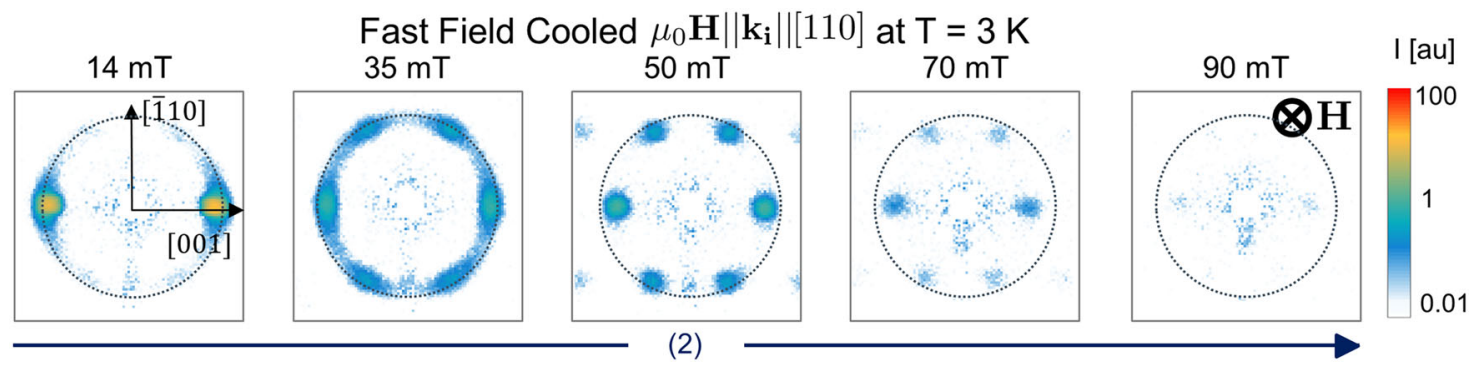

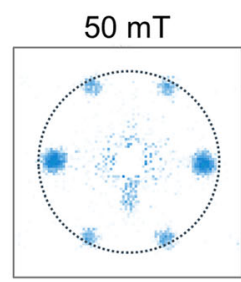

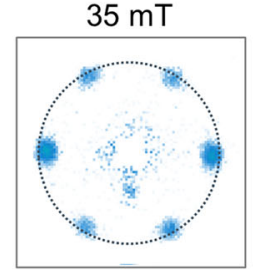

(3)

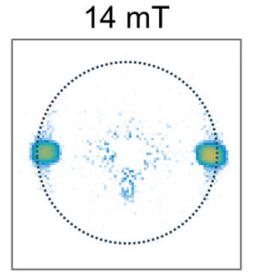

Zero Fielc Zero Fiel
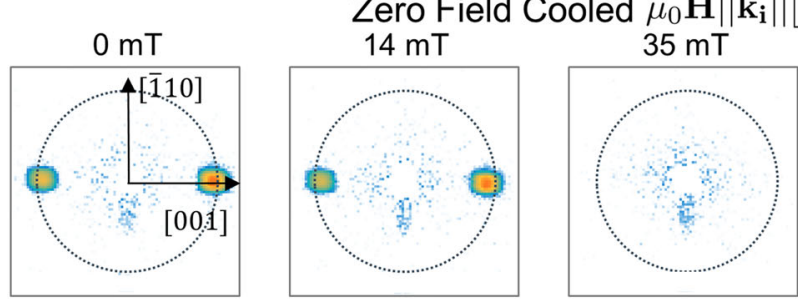
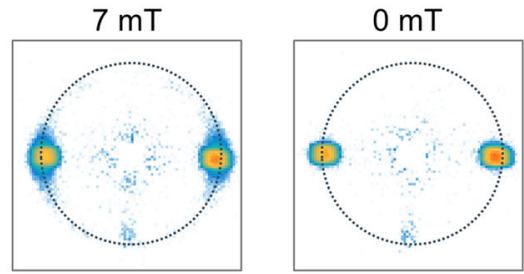

(5)
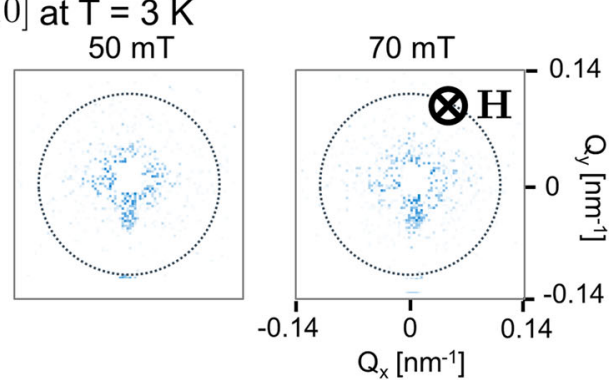

Fig. 3 SANS patterns recorded at $T=3 \mathrm{~K}$ and with the magnetic field applied along the [110] crystallographic direction.The magnetic field was applied parallel to the incoming neutron beam $\left(\mathbf{H}|| \mathbf{k}_{\mathrm{i}}\right)$ following the fast field cooled (FFC) and zero field cooled protocols. The arrows and numbers below the SANS patterns indicate the step of the FFC protocol, as defined in Fig. 1, for which the measurements were performed

neutron beam wavevector $\mathbf{k}_{\mathbf{i}}$. In the following we will focus on the results obtained in the latter configuration, which is the one probing skyrmionic correlations. Representative results for $\mathbf{H} \perp \mathbf{k}_{\mathbf{i}}$ are provided in the Figures S13-S15.

Figure 2 shows a representative set of SANS patterns at $T=3 \mathrm{~K}$ after FFC, for $\mathbf{H} \|[111]$. A ring of scattering appears at $\mu_{0} H=14 \mathrm{mT}$, which evolves with increasing magnetic field into a weak pattern with the six-fold symmetry characteristic of SkL scattering. ${ }^{8}$ By further increasing the magnetic field, this scattering weakens and persists up to the $\mu_{0} H_{C 2}$ transition line. At higher magnetic fields, the scattering vanishes, implying that the SkLs have dissolved. Surprisingly, when the magnetic field is subsequently decreased from $\mu_{0} H=90$ to $0 \mathrm{mT}$, the scattering patterns with six-fold symmetry reappear for $65 \mathrm{mT}>\mu_{0} H>7 \mathrm{mT}$. This is a remarkable result because it implies that a memory of the SkL phase persists at magnetic fields high enough to suppress not only the SkL scattering but also any spiral modulations. Additional measurements indicate that the SkL scattering reappears even after applying a field of $200 \mathrm{mT}$, i.e. more than twice as large as $\mu_{0} H_{\mathrm{C} 2}$. Thus, the memory of skyrmionic correlations is extremely robust and can only be erased by (relatively) high magnetic fields. This result is even more remarkable if one considers that these robust skyrmions are metastable, as they were induced by the FFC procedure. Indeed, this scattering disappears once the field has been reduced to zero, and it is not observed by FFC under magnetic fields exceeding the lower and upper boundaries of the A-phase (Fig. S4) or when the sample is field cooled at a much slower cooling rate of $10 \mathrm{~K} / \mathrm{min}$, or zero-field cooled (Fig. 2). Skyrmions may survive due to magnetic field inhomogeneities produced by stray fields, in particular near the sample edges, or in the form of magnetic defects such as torons. ${ }^{18,45-47}$
A similar behavior is found for $\mathbf{H} \|[110]$, as evidenced by the SANS patterns shown in Fig. 3. After FFC at $\mu_{0} H=14 \mathrm{mT}$, the scattering pattern mainly consists of two helical peaks along the [001] direction-a metastable spiral domain with the wave vector perpendicular to the field. This scattering is superimposed on a very weak additional signal, which evolves to a clear SkL pattern at $\mu_{0} H=35 \mathrm{mT}$, i.e. a field where the helical peaks have almost completely disappeared. With increasing magnetic field, the SkL scattering weakens and finally disappears at $\mu_{0} \mathrm{H}=85 \mathrm{mT}$. When the magnetic field is subsequently decreased from $\mu_{0} H=90$ to $0 \mathrm{mT}$, the SkL scattering reappears around $50 \mathrm{mT}$, albeit with a much lower intensity and within a smaller magnetic field interval than for $\mathbf{H} \|[111]$ (Fig. S3).

The most intriguing behavior is found for $\mathbf{H} \|[001]$, the field direction for which the tilted conical phase appears close to $\mu_{0} H_{C 2}{ }^{37}$ After FFC, the SANS pattern at $14 \mathrm{mT}$, shown in Fig. 4, reveals four Bragg peaks originating from the metastable spiral domains along [001] and [010] that are in the scattering plane. In addition, an intense ring of scattering with radius $\tau=2 \pi / \ell$ is visible, $\ell$ being the pitch of a helical modulation. One can distinguish 12 peaks originating from two SkL domains oriented along [001] and [010], as is also the case in the A-phase. ${ }^{48}$ With increasing magnetic field, the helical peaks fade away, whereas the ring of scattering gains intensity, broadens and its radius decreases considerably. This ring-like scattering is due to skyrmionic correlations because it is concentrated on the plane perpendicular to the field as evidenced by the SANS patterns for $\mathbf{H} \perp \mathbf{k}_{\mathrm{i}}$ shown in Fig. S15. The ring disappears above $\mu_{0} H \approx 70 \mathrm{mT}$ and when it reappears by decreasing the magnetic field from 90 to $0 \mathrm{mT}$, its broadening is even more pronounced. This ring is also seen in the ZFC patterns although the intensity is much lower after ZFC than after FFC (see also Figs. S4 and S5). However, the 

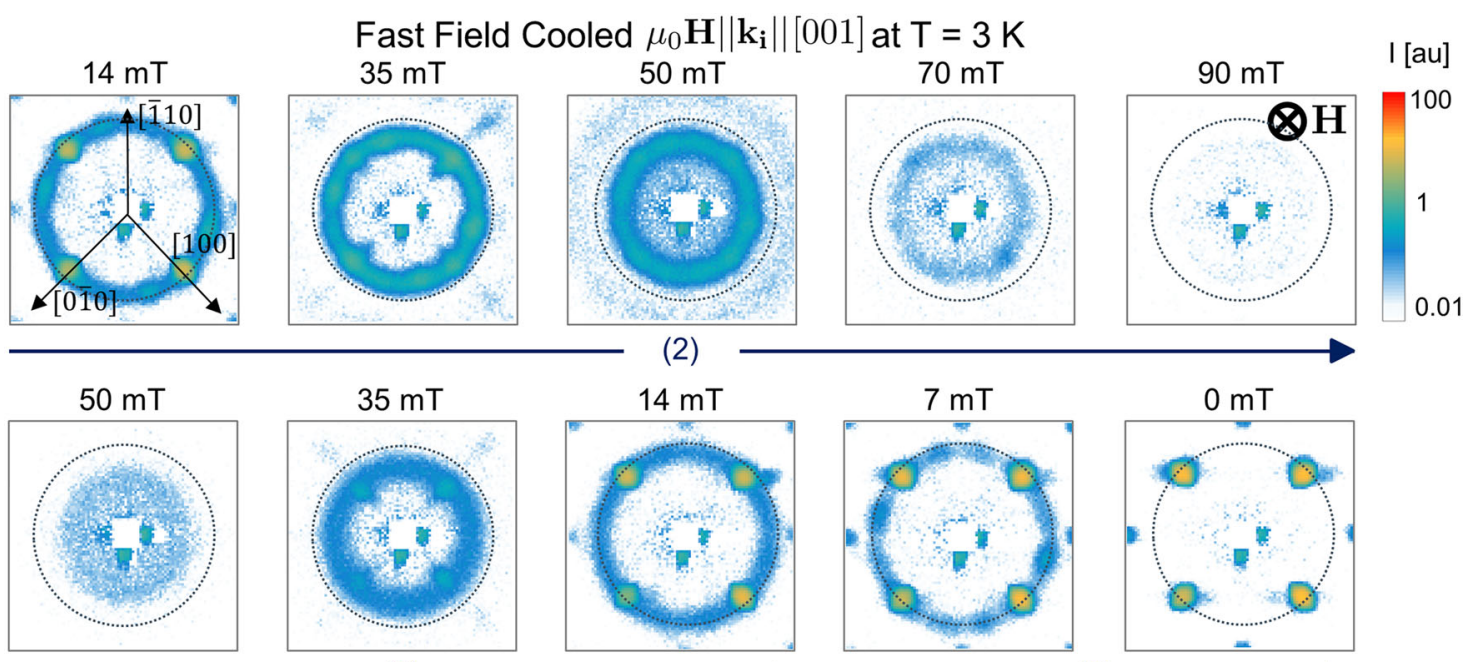

(3)

(5)
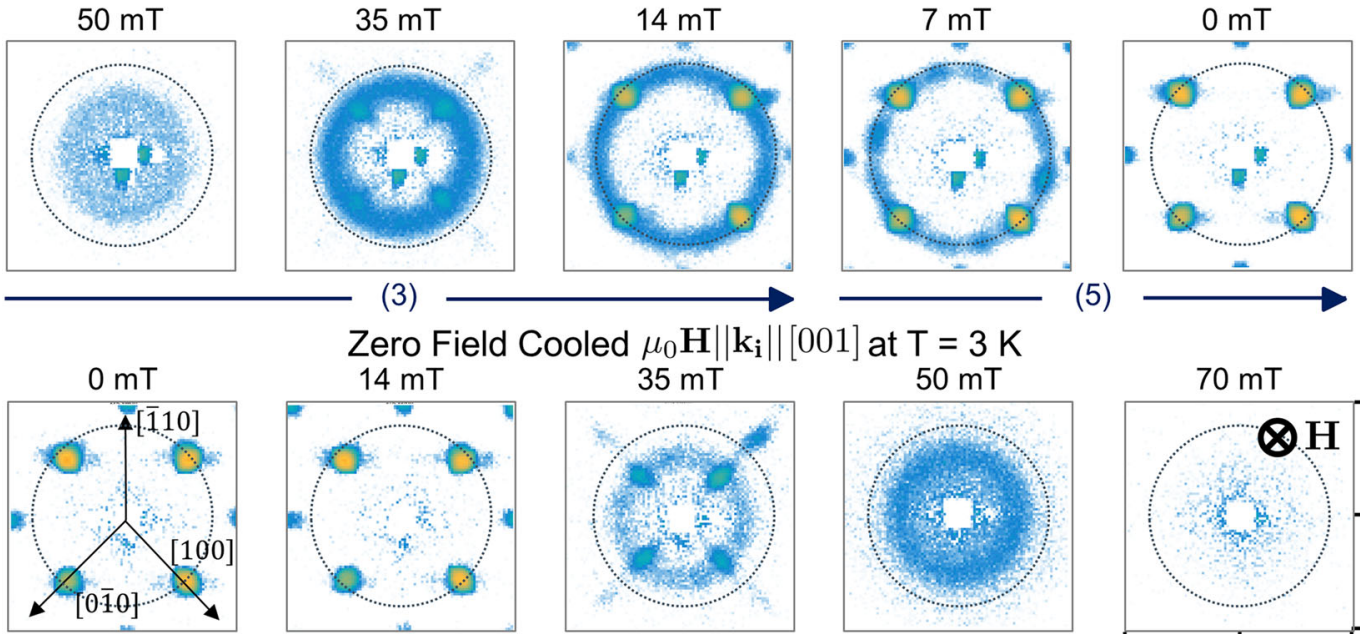

Zero Field
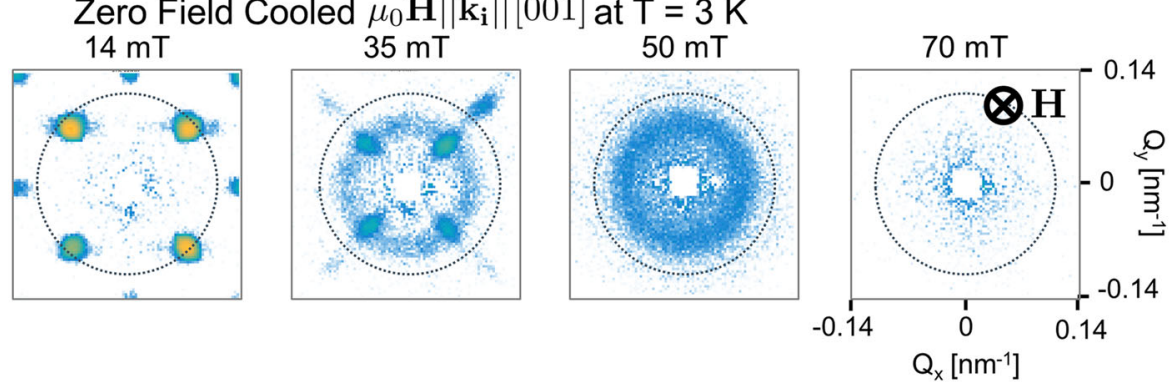

Fig. 4 SANS patterns recorded at $T=3 \mathrm{~K}$ and with the magnetic field applied along the [001] crystallographic direction. The magnetic field was applied parallel to the incoming neutron beam $\left(\mathbf{H} \| \mathbf{k}_{i}\right)$ following the fast field cooled (FFC) and zero field cooled protocols. The arrows and numbers below the SANS patterns indicate the step of the FFC protocol, as defined in Fig. 1, for which the measurements were performed
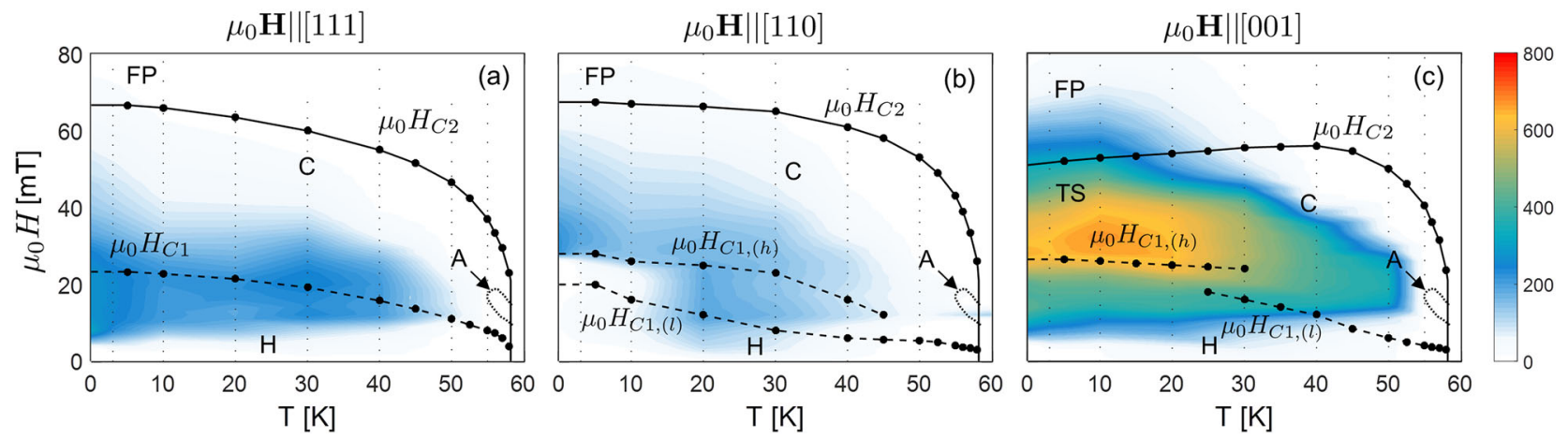

Fig. 5 Phase diagrams indicating the extent and intensity of skyrmionic scattering after FFC through the A-phase. Contour plots of the total neutron scattering outside the helical peaks measured following steps 2 and 5 of Fig. 1. The magnetic field was applied parallel to the incoming neutron beam $\left(\mathbf{H} \mid \mathbf{k}_{\mathbf{i}}\right)$ and along the $\mathbf{a}$ [111], $\mathbf{b}$ [110], and $\mathbf{c}$ [001] crystallographic directions and averaged using the masks shown in Fig. S1 of the Supplementary Information. The lower $\left(\mu_{0} H_{\mathrm{C} 1,(\mathrm{l})}\right)$ and upper $\left(\mu_{0} H_{\mathrm{C} 1,(\mathrm{~h})}\right)$ helical-to-conical, conical-to-field polarized $\left(\mu_{0} H_{\mathrm{C} 2}\right)$ transition lines, as well as the boundaries of the A-phase just below $T_{C}$ have been derived from susceptibility measurements. ${ }^{37}$ The letters $A$, $H$, C, TS, and FP denote the A-phase, helical, conical, tilted spiral, and field polarized phases, respectively

radius and widths of the rings after FFC and ZFC are the same, which implies that they have the same origin. We thus conclude, that these rings arise from thermodynamically stable skyrmionic correlations which coexist with the conical and tilted spiral phases.

The experimental results are summarized in Figs. 5 and 6, which show contour plots of the total scattering recorded after FFC (steps (2) and (5) in Fig. 1) and ZFC (steps (2) and (3) in Fig. S6), respectively. The intensity has been obtained by excluding the helical and conical peaks, as explained in the Supplementary Information, and corresponds to the total scattering that can be attributed to skyrmionic correlations. This scattering appears over large areas, the extent of which strongly depends on the specific orientation of the magnetic field with respect to the crystallographic lattice. Remarkably, after FFC the skyrmionic scattering appears around the $H_{C 1}$ line for $\mathbf{H} \|[111]$ and $\mathbf{H} \|[110]$, whereas for $\mathbf{H} \|[001]$ it spreads from below $H_{\mathrm{C} 1}$ to above $H_{\mathrm{C} 2}$. We note that this metastable skyrmionic scattering is reproducible and does not decay within the time scale of our SANS experiments.

The low-temperature skyrmionic scattering seen after ZFC for $\mathbf{H} \|[001]$ shows a clear hysteretic behavior, appearing around the $H_{\mathrm{C} 2}$ line when the magnetic field increases from zero to above $H_{\mathrm{C} 2}$ (step (2) in Fig. S6), and around the $H_{\mathrm{C} 1}$ line when the magnetic 


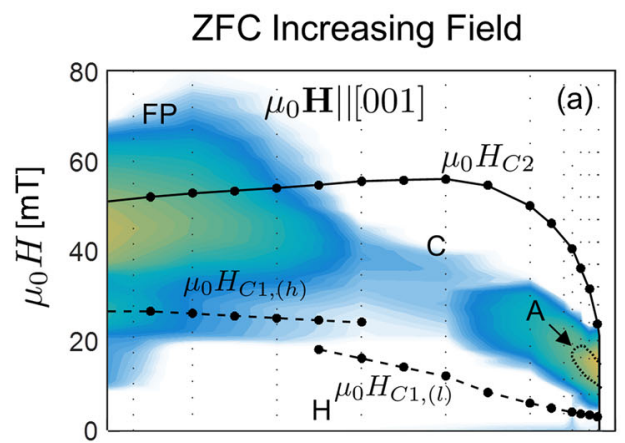

\section{ZFC Decreasing Field}
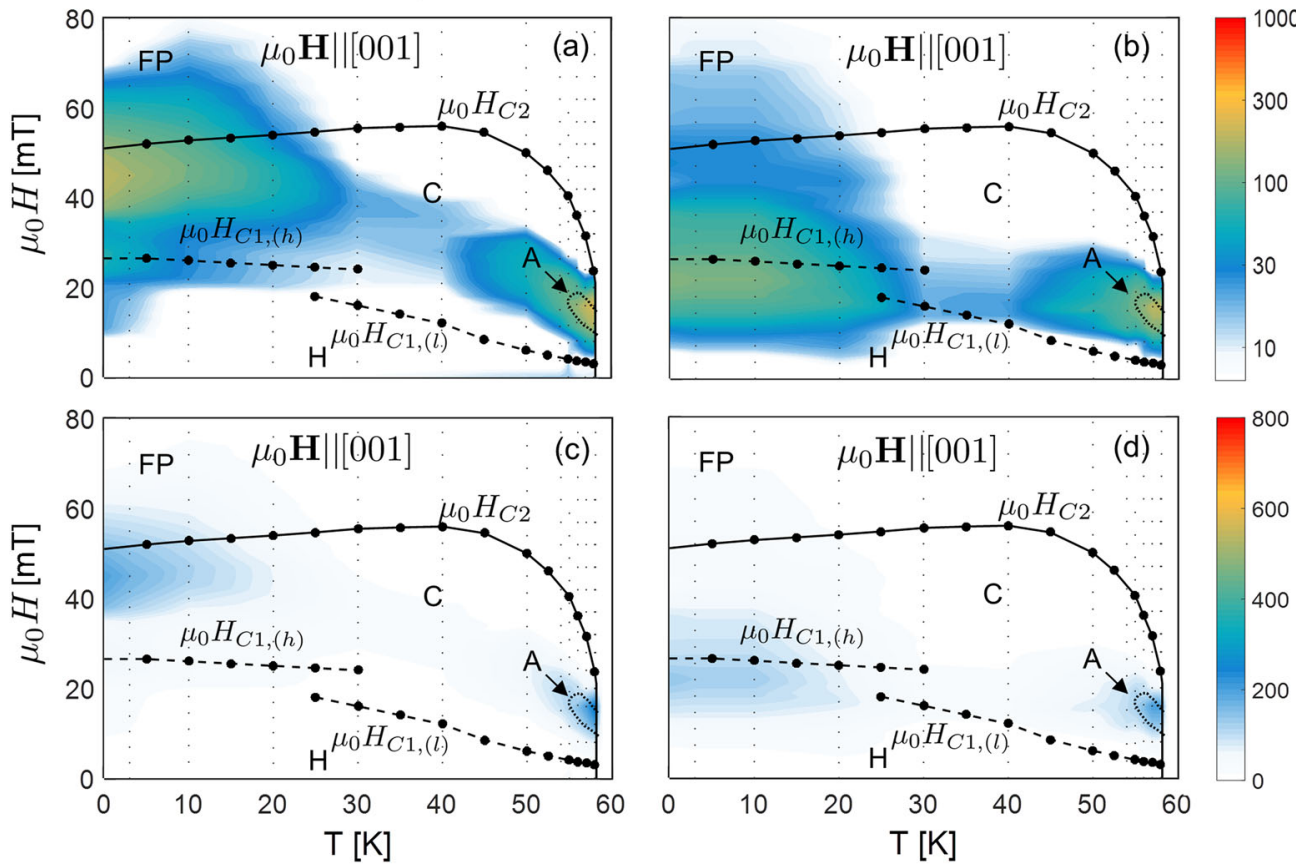

Fig. 6 Phase diagrams indicating the extent and intensity of skyrmionic scattering after zero field cooling (ZFC) for $\mathbf{H}\left\|\mathbf{k}_{\mathbf{i}}\right\|[001]$. The measurements were performed upon increasing the magnetic field, step (2) in Fig. S6 (panels a and c) and decreasing the magnetic field, step (3) in Fig. S6 (panels $\mathbf{b}$ and $\mathbf{d}$ ). The contour plots of panels $\mathbf{a}$ and $\mathbf{b}$ use a logarithmic color scale, whereas those of panels $\mathbf{c}$ and $\mathbf{d}$ a linear one

field subsequently decreases from above $H_{\mathrm{C} 2}$ to zero (step (3) in Fig. S6). These results provide hints to the underlying skyrmion nucleation and stabilization mechanisms discussed below.

The high quality of our neutron scattering data also provides new insights on the low-temperature skyrmionic correlations observed after ZFC for $\mathbf{H} \|[001]$. As revealed by the logarithmic scale of Fig. $6 a$, b, this low-temperature skyrmionic scattering is not confined to pockets, as previously suggested, ${ }^{38}$ but extends up to the A-phase. Remarkably, the hysteretic behavior sets in at temperatures just below the A-phase, with one branch following the $H_{\mathrm{C} 1}$ line over the whole phase diagram and the other one reaching the $H_{C 2}$ line at low temperatures.

\section{DISCUSSION}

In order to understand the large differences between the contour plots shown in Figs. 5 and 6, as well as the underlying skyrmion nucleation and stabilization mechanisms, we consider the Dzyaloshinskii model of cubic non-centrosymmetric ferromagnets. ${ }^{26,27,49}$ This model includes the ferromagnetic exchange, DM, and Zeeman interaction energies, i.e. the principal interactions essential to stabilize modulated states:

$w=(\operatorname{grad} \mathbf{m})^{2}+\mathbf{m} \cdot \operatorname{rot} \mathbf{m}-\mu_{0} M \mathbf{m} \cdot \mathrm{h}$.

Here, distances are measured in units of $\mathbf{x}=\mathbf{r} / \ell$ and energy is measured in units of $D^{2} / A, A$ and $D$ being the exchange stiffness and Dzyaloshinskii constants, respectively. $\mathbf{m}$ is the unit vector in the direction of the magnetization, $\mathbf{M}=\mathbf{m} M$, and $\mathbf{h}=\mathbf{H} A / D^{2}$.

While for low-symmetry classes, such as $C_{\mathrm{nv}}{ }^{50}$ and $D_{2 \mathrm{~d}}$, the DM interaction alone stabilizes $S k L s,{ }^{51}$ for cubic helimagnets, additional terms are required to lower the energy of the skyrmion lattice with respect to the conical spiral state. We consider anisotropic contributions that include cubic anisotropy with $k_{\mathrm{c}}>0$ (easy $\langle 001\rangle$ axes) and exchange anisotropy with $b_{\mathrm{EA}}<0$ (easy
〈111〉 axes):

$$
\begin{aligned}
w_{\mathrm{a}}=k_{\mathrm{c}} & \left(m_{x}^{2} m_{y}^{2}+m_{x}^{2} m_{z}^{2}+m_{y}^{2} m_{z}^{2}\right) \\
& +b_{\mathrm{EA}}\left(\left(\partial_{x} m_{x}\right)^{2}+\left(\partial_{y} m_{y}\right)^{2}+\left(\partial_{z} m_{z}\right)^{2}\right) .
\end{aligned}
$$

SkLs that have a lower energy than other modulated states can only be found for $\mathbf{H} \|[001]$. This explains why the skyrmionic correlations occur after ZFC only for $\mathbf{H} \|[001]$ (Fig. 4) and why they are the strongest after FFC (Fig. 5c).

Figure $7 \mathrm{a}$ shows the zero-temperature phase diagram in the $\left(k_{\mathrm{c}}\right.$ $h)$ plane calculated for zero exchange anisotropy, which contains the conical spiral state with the propagation vector $\mathbf{q}\|\mathbf{H}\|[001]$ (blue color), the skyrmion lattice phase (pink color) and the field polarized phase (white color). The SkL has the lowest energy for relatively small values of $k_{c} \gtrsim 0.04$ and is metastable in a much wider region of the phase diagram. The dotted line in Fig. 7a marks the magnetic field at which the energy of the SkL for a given $k_{\mathrm{c}}$ becomes equal to the energy of the metastable helical spiral states with the wave vectors along the [100] and [010] directions. One expects that when $h$ becomes higher than this field, metastable spirals rupture forming pairs of merons, each with a topological charge $1 / 2 .{ }^{52,53}$ Merons, which may also nucleate at the boundary between the metastable spiral and conical domains, ultimately evolve into skyrmions at higher magnetic fields. Since the SkL phase remains metastable below the dashed line, one expects skyrmionic states to persist for fields lower than $h=0.11$, until they undergo an elliptical instability into spiral states, ${ }^{54}$ which explains the hysteresis effect shown in Fig. 6.

Above the line separating the SkL from the field polarized state, skyrmions remain stable but the skyrmion lattice disappears because of the repulsion between skyrmions. In this way, it is possible to reconcile the disappearance of the $\mathrm{SkL}$ signal upon increasing the field above $\sim \mu_{0} H_{\mathrm{C} 2}$ and its surprising comeback when the field is lowered from a value greatly exceeding $\mu_{0} H_{\mathrm{C} 2}$. The disappearance of the skyrmion lattice which does not destroy all skyrmions resembles sublimation of a solid. The (meta)stability of isolated skyrmions can be enhanced by stray fields, grain 
(a)

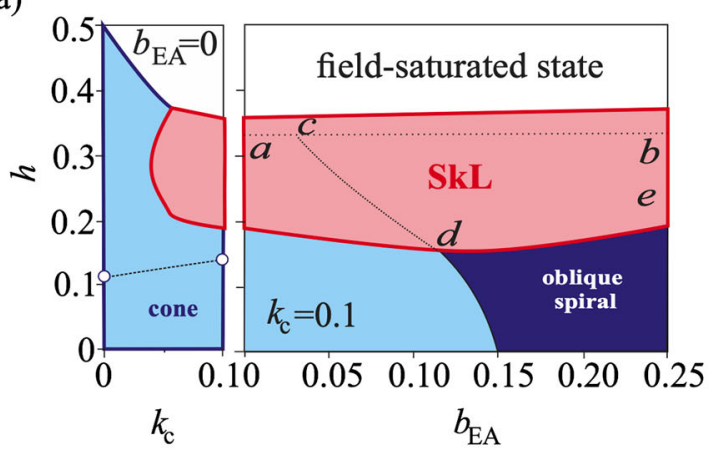

(b)

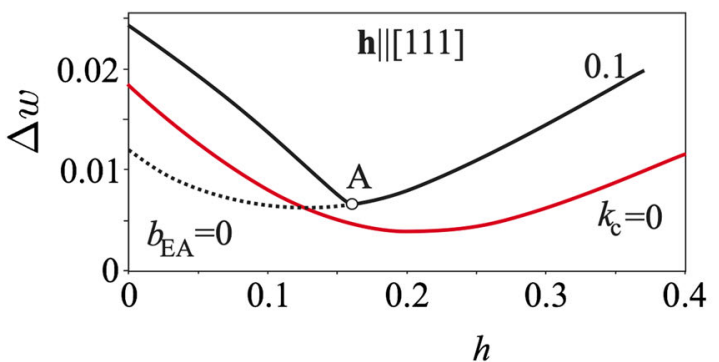

(c)

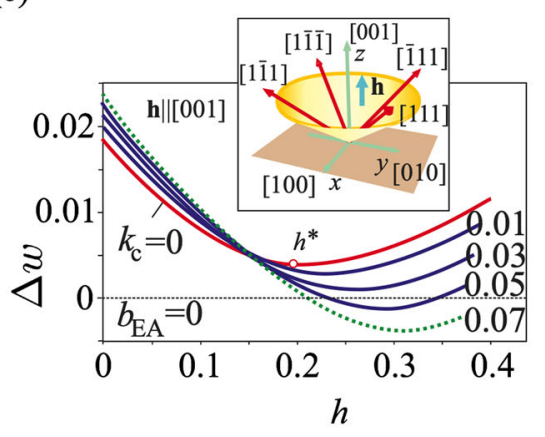

(d)

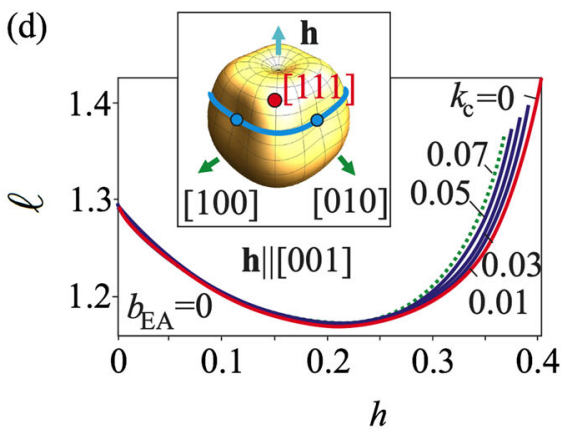

Fig. 7 Stable and metastable skyrmion lattice states at zero temperature. a Zero-temperature phase diagrams with the magnetic field applied along [001], where $h$ is the dimensionless field magnitude defined in the text, vs. the cubic anisotropy strength, $k_{c}$, for zero exchange anisotropy, $b_{\mathrm{EA}}=0$, (left panel) and vs. $b_{\mathrm{EA}}$, for $k_{\mathrm{c}}=0.1$ (right panel). $\mathbf{b}$ and $\mathbf{c}$ Difference between the energy densities of the skyrmion lattice and conical (or helical) spiral states, $\Delta w$, plotted as a function of $h$ applied along [111] and [001], respectively, for specific values of the cubic anisotropy. If the magnetic field is applied along [111], $\Delta w$ is always positive, thus the skyrmion lattice is energetically unfavorable. $\Delta w$ goes though a minimum, which shifts towards low fields with increasing anisotropy (black dotted line). Moreover, at low fields the helical spiral states have lower energy than the conical phase (solid black line). If the magnetic field is applied along [001], the tendency is quite the opposite and for smaller $k_{\mathrm{c}}$ the stability region of the $S k L$ state $(\Delta w<0)$ lies within the conical spiral phase. For larger values of $k_{\mathrm{c}}$, this region shifts towards higher fields, and at the upper boundary the SkL undergoes a first-order transition to the field polarized state. The inset shows a sketch of the magnetization rotation in the conical phase in the presence of cubic anisotropy with hard $\langle 111\rangle$ (red arrows) and easy $\langle 100\rangle$ (green arrows) axes. d Field dependence of the SkL pitch $\ell$. The curves, that are in qualitative agreement with the experimental results (Fig. S11), are terminated at the transition to the field polarized state for all considered values of $k_{\mathrm{c}}$. For $k_{\mathrm{c}}=0, \ell$ diverges at this transition (red line). The upper magnetic field limit for $\ell$ decreases with increasing $k_{\mathrm{c}}$ as lower fields are needed to release isolated skyrmions. The inset shows the three-dimensional polar plot of the conical spiral state energy density as a function of the wave vector direction. The path of the rotating magnetization in the conical phase is imaged by the blue line with blue circles being the minima for the homogeneous state

boundaries, and formation of skyrmion bags also considered in the context of chiral liquid crystals. ${ }^{55}$

Figure 7a shows the effect of exchange anisotropy on the zero temperature phase diagram, which for $b_{\mathrm{EA}}>b_{\mathrm{EA}}^{*}$ transforms the conical spiral state with $\mathbf{q} \| \mathbf{H}$ into the tilted spiral state (dark blue color), in which the wave vector tilts towards one of the nearest four body-diagonal $\langle 111\rangle$ directions. ${ }^{37}$ The value of $b_{\mathrm{EA}}$ cannot be much larger than $b_{\mathrm{EA}}^{*}$, because otherwise the tilted spiral state would extend down to zero magnetic field, which is inconsistent with the experimental results. In the b-c-d-e region the tilted spiral state is locally stable but has a higher energy than the SkL phase. We note that neither cubic nor exchange anisotropy stabilizes a square $\mathrm{SkL}$, in agreement with the experimental results.

The SkL is metastable for the [111] and [110] directions explaining the lower FFC scattering intensities and the absence of a ZFC skyrmion signal (Fig. 5a, b). Yet, also for these directions, the SkL scattering is the strongest along the helical-to-conical transition line, which is also the field region where the energy difference between the SkL and spiral states is the smallest (Fig. 7b). In addition, the boundaries between the metastable SkL, helical and conical phases catalyze the appearance of topological charge. Indeed, the nontrivial topology of magnetic skyrmions gives rise to energy barriers hindering their creation and destruction, which makes transitions between topological and non-topological magnetic states different from, e.g. the water-ice transition where both phases are composed of the same building blocks (water molecules).

Furthermore, the intensity of the skyrmion lattice scattering for $\mathbf{H} \|[111]$ shows a complex temperature dependence: at a constant magnetic field of, e.g. $\mu_{0} \mathrm{H}=20 \mathrm{mT}$, the intensity first increases, goes through a maximum at $\sim 30 \mathrm{~K}$, then decreases to a minimum at $\sim 15 \mathrm{~K}$, before increasing again at lower temperatures (see Fig. S7). This complex temperature dependence might be related to different orientations of the metastable skyrmion lattice. Our calculations show that in low magnetic fields the spatial orientation of the metastable skyrmion lattice is determined by magnetic anisotropies and the basis vectors of the skyrmion crystal are not necessarily orthogonal to the applied field. The situation is similar to the temperature dependence of the wave vector of the tilted spiral state: Its deviation from the magnetic field direction decreases with temperature, which can be interpreted as the result of the competition between the temperature-dependent cubic and exchange anisotropies. For dominant cubic anisotropy the skyrmion tubes in the metastable SkL may be normal to the (001) planes, while the relatively large exchange anisotropy may re-orient the tubes along the $\langle 111\rangle$ directions. The temperature dependence of the SkL intensity along the $\mu_{0} H_{\mathrm{C} 1}$ line is even more pronounced for $\mathbf{H} \|[110]$. 
In conclusion, extremely robust low-temperature skyrmionic states can be induced for all field directions and over large areas of the phase diagram. These skyrmionic states are stabilized by competing cubic and exchange anisotropies, and are thermodynamically stable when the magnetic field is applied along the [001] axis. The formation and destruction of these skyrmionic states is enhanced by nascent and disappearing spiral states near critical lines which catalyze topological charge changing processes. In particular, at low magnetic fields, the spatial orientation of the skyrmion lattice is determined by magnetic anisotropies and is not necessarily orthogonal to the applied field. On the other hand, these skyrmions are surprisingly resilient to high magnetic fields and their memory persists, possibly in the form of isolated skyrmions or torons, even in the field polarized state. These findings underscore the paramount role of magnetic anisotropies in stabilizing novel skyrmionic states and provide valuable directions to manipulate and tune skyrmions for future applications, possibly with electric fields or strain.

\section{METHODS}

$\mathrm{Cu}_{2} \mathrm{OSeO}_{3}$ has first been synthesized by a reaction of $\mathrm{CuO}$ (Alfa Aesar 99.995\%) and $\mathrm{SeO}_{2}$ (Alfa Aesar 99.999\%) at $300^{\circ} \mathrm{C}$ (2 days) and $600{ }^{\circ} \mathrm{C}$ (7 days) in evacuated fused silica tubes. Starting from this microcrystalline powder, the $3 \times 3 \times 4 \mathrm{~mm}^{3} \mathrm{Cu}_{2} \mathrm{OSeO}_{3}$ single crystal was grown by a chemical transport reaction in a temperature gradient from $580^{\circ} \mathrm{C}$ (source) to $460^{\circ} \mathrm{C}$ (sink), and a transport additive concentration of $1 \mathrm{mg} / \mathrm{cm}^{3} \mathrm{NH}_{4} \mathrm{Cl}$ (23 mg) (Alfa Aesar 99.999\%). The sample was the same as the one used in our previous work. ${ }^{37,48}$ It was glued to an aluminum cylinder with a diameter of $1 \mathrm{~mm}$ around which $1 \mathrm{~m}$ constantan wire was wound with a total resistance of $30 \Omega$. The wire was glued with GE low-temperature varnish and was used to generate large heat pulses.

The experiments were performed at the SANS instrument D33 of the Institut Laue-Langevin ${ }^{56}$ with an incident wavelength of $0.8 \mathrm{~nm}$, a sample-to-detector distance of $12.8 \mathrm{~m}$, and a wave-vector transfer resolution of $\Delta \lambda / \lambda=10 \%$. The sample was placed inside an Oxford Instruments $7 \mathrm{~T}$ horizontal-field cryomagnet, the remanent field of which did not exceed some $10^{-4} \mathrm{~T}$. The constantan heater was connected to an external power supply. Fast cooling was performed by first stabilizing the sample at the temperature of interest. Subsequently, a current of $0.3 \mathrm{~A}$ was applied for $1.5 \mathrm{~s}$ to the constantan heater, providing sufficiently heat to bring the sample to $T \gg T_{\mathrm{C}}$. Switching off the current resulted in a rapid cooling with an average cooling rate of $10 \mathrm{~K} / \mathrm{s}$, as estimated from a comparison of the scattered intensity of the SANS patterns at zero magnetic field (recorded every $0.8 \mathrm{~s}$ ) with the temperature-dependent evolution of the scattered intensity of the helical Bragg peaks at zero magnetic field. All magnetic fields are corrected for demagnetization effects assuming a demagnetization factor of $1 / 3$. The reader is referred to the Supplementary Information (Figs. S1, S2 and S8-S12) for details on the data analysis and treatment.

\section{DATA AVAILABILITY}

All data needed to evaluate the conclusions in the paper are included in the paper and/or the Supplementary Information. The raw experimental data is available at doi. org/10.5291/ILL-DATA5-42-461. Additional data related to this paper may be requested from L.J.B. and C.P. (I.j.bannenberg@tudelft.nl and c.pappas@tudelft.nl).

\section{ACKNOWLEDGEMENTS}

The authors wish to thank the ILL support staff for their assistance and are grateful for the kind help of B. Ouladdiaf with aligning the single crystals at OrientExpress. The work of L.J.B. and C.P. is financially supported by The Netherlands Organization for Scientific Research through project 721.012.102 (LARMOR). C.P. and M.M. acknowledge Vrije FOM-programma 'Skyrmionics'. A.O.L. acknowledges JSPS Core-to-Core Program, Advanced Research Networks (Japan) and JSPS Grant-in-Aid for Research Activity Start-up 17H06889.

\section{AUTHOR CONTRIBUTIONS}

L.J.B., H.W., E.L.-B., A.L., and C.P. performed the neutron scattering experiments with the support of R.C. L.J.B. performed the analysis of the experimental data. M.P.S. provided the sample. A.O.L. and M.M. developed the theoretical approach. The manuscript was written by L.J.B., A.O.L., M.M. and C.P. with input from the other coauthors.

\section{ADDITIONAL INFORMATION}

Supplementary information accompanies the paper on the npj Quantum Materials website (https://doi.org/10.1038/s41535-019-0150-7).

Competing interests: The authors declare no competing interests.

Publisher's note: Springer Nature remains neutral with regard to jurisdictional claims in published maps and institutional affiliations.

\section{REFERENCES}

1. Skyrme, T. H. R. A non-linear field theory. Proc. R. Soc. Lond. A 260, 127-138 (1961).

2. Rho, M. \& Zahed, I. The Multifaceted Skyrmion. 2nd ed (World Scientific, Singapore, 2016).

3. Callan, C. G. Jr. \& Witten, E. Monopole catalysis of skyrmion decay. Nucl. Phys. B 239, 161-176 (1984).

4. Al Khawaja, U. \& Stoof, H. Skyrmions in a ferromagnetic Bose-Einstein condensate. Nature 411, 918-920 (2001).

5. Fukuda, J.-I. \& Žumer, S. Quasi-two-dimensional Skyrmion lattices in a chiral nematic liquid crystal. Nat. Commun. 2, 246 (2011).

6. Ackerman, P. J., Boyle, T. \& Smalyukh, I. I. Squirming motion of baby skyrmions in nematic fluids. Nat. Commun. 8, 673 (2017).

7. Tsesses, S. et al. Optical skyrmion lattice in evanescent electromagnetic fields. Science 15, eaau0227 (2018).

8. Mühlbauer, S. et al. Skyrmion lattice in a chiral magnet. Science 323, 915-919 (2009).

9. $\mathrm{Yu}, \mathrm{X}$. Z. et al. Real-space observation of a two-dimensional skyrmion crystal. Nature 465, 901-904 (2010).

10. $\mathrm{Yu}, \mathrm{X}$. Z. et al. Skyrmion flow near room temperature in an ultralow current density. Nat. Commun. 3, 988 (2012).

11. Fert, A., Cros, V. \& Sampaio, J. Skyrmions on the track. Nat. Nanotech. 8, 152-156 (2013).

12. Nagaosa, N. \& Tokura, Y. Topological properties and dynamics of magnetic skyrmions. Nat. Nanotech. 8, 899-911 (2013).

13. White, J. S. et al. Electric-field induced skyrmion distortion and giant lattice rotation in the magnetoelectric insulator $\mathrm{Cu}_{2} \mathrm{OSeO}_{3}$. Phys. Rev. Lett. 113, 107203 (2014).

14. Fert, A., Reyren, N. \& Cros, V. Magnetic skyrmions: advances in physics and potential applications. Nat. Mater. 2, 201731 (2017).

15. Wilson, M. N., Butenko, A. B., Bogdanov, A. N. \& Monchesky, T. L. Chiral skyrmions in cubic helimagnet films: the role of uniaxial anisotropy. Phys. Rev. B 89, 094411 (2014).

16. Buhrandt, S. \& Fritz, L. Skyrmion lattice phase in three-dimensional chiral magnets from Monte Carlo simulations. Phys. Rev. B 88, 195137 (2013).

17. Wilhelm, $\mathrm{H}$. et al. Precursor phenomena at the magnetic ordering of the cubic helimagnet FeGe. Phys. Rev. Lett. 107, 127203 (2011).

18. Leonov, A. O. \& Inoue, K. Homogeneous and heterogeneous nucleation of skyrmions in thin layers of cubic helimagnets. Phys. Rev. B 98, 054404 (2018).

19. Levatić, I. et al. Dramatic pressure-driven enhancement of bulk skyrmion stability. Sci. Rep. 6, 21347 (2016).

20. Okamura, Y., Kagawa, F., Seki, S. \& Tokura, Y. Transition to and from the skyrmion lattice phase by electric fields in a magnetoelectric compound. Nat. Commun. 7, 12669 (2016).

21. Kruchkov, A. J. et al. Direct electric field control of the skyrmion phase in a magnetoelectric insulator. Sci. Rep. 8, 10466 (2018).

22. White, J. S. et al. Electric field driven topological phase switching and skyrmion lattice metastability in magnetoelectric $\mathrm{Cu}_{2} \mathrm{OSeO}_{3}$. Phys. Rev. Appl. 10, 014021 (2018).

23. $\mathrm{Wu}, \mathrm{H}$. C. et al. Unexpected observation of splitting of skyrmion phase in $\mathrm{Zn}$ doped $\mathrm{Cu}_{2} \mathrm{OSeO}_{3}$. Sci. Rep. 5, 13579 (2015).

24. Seki, S. et al. Stabilization of magnetic skyrmions by uniaxial tensile strain. Phys. Rev. B 96, 220404 (2017).

25. Nakajima, T. et al. Uniaxial-stress effects on helimagnetic orders and skyrmion lattice in $\mathrm{Cu}_{2} \mathrm{OSeO}_{3}$. J. Phys. Soc. Jpn. 87, 094709 (2018).

26. Dzyaloshinskii, I. E. Theory of helicoidal structures in antiferromagnets.1. Nonmetals. Sov. Phys. JETP 19, 960 (1964).

27. Dzyaloshinskii, I. E. The theory of helicoidal structures in antiferromagnets. III. metals. Sov. Phys. JETP 20, 665 (1965). 
28. Bogdanov, A. N. \& Yablonskii, D. A. Contribution to the theory of inhomogeneous states of magnets in the region of magnetic-field induced phase transitions. Mixed state of antiferromagnets. Sov. Phys. JETP 69, 142 (1989).

29. Bogdanov, A. N. \& Hubert, A. The stability of vortex-like structures in uniaxial ferromagnets. J. Magn. Magn. Mater. 195, 182 (1999).

30. Rößler, U. K., Bogdanov, A. N. \& Pfleiderer, C. Spontaneous skyrmion ground states in magnetic metals. Nature 442, 797-801 (2006).

31. Leonov, A. O. Twisted, Localized, and Modulated States Described in the Phenomenological Theory of Chiral and Nanoscale Ferromagnets. Ph.D. thesis, Technical University of Dresden (2012).

32. Butenko, A. B., Leonov, A. A., Rößler, U. K. \& Bogdanov, A. N. Stabilization of skyrmion textures by uniaxial distortions in noncentrosymmetric cubic helimagnets. Phys. Rev. B 82, 052403 (2010).

33. Jiang, W. et al. Skyrmions in magnetic multilayers. Phys. Rep. 704, 1-49 (2017).

34. Leonov, A. O. et al. Chiral surface twists and skyrmion stability in nanolayers of cubic helimagnets. Phys. Rev. Lett. 117, 087202 (2016).

35. McGrouther, D. et al. Internal struc ture of hexagonal skyrmion lattices in cubic helimagnets. New J. Phys. 18, 095004 (2016).

36. Zhang, S. L., van der Laan, G., Wang, W. W., Haghighirad, A. A. \& Hesjedal, T. Direct observation of twisted surface skyrmions in bulk crystals. Phys. Rev. Lett. 120, 227202 (2018).

37. Qian, F. et al. New magnetic phase of the chiral skyrmion material $\mathrm{Cu}_{2} \mathrm{OSeO}_{3}$. Sci. Adv. 4, eaat7323 (2018).

38. Chacon, A. et al. Observation of two inde pendent skyrmion phases in a chiral magnetic material. Nat. Phys. 14, 936 (2018).

39. Janson, $\mathrm{O}$. et al. The quantum nature of skyrmions and half-skyrmions in $\mathrm{Cu}_{2} \mathrm{O}-$ $\mathrm{SeO}_{3}$. Nat. Commun. 5, 5376 (2014).

40. Oike, $\mathrm{H}$. et al. Interplay between topological and thermodynamic stability in a metastable magnetic skyrmion lattice. Nat. Phys. 12, 62-66 (2016).

41. Bannenberg, L. J. et al. Ex-tended skyrmion lattice scattering and long-time memory in the chiral magnet $\mathrm{Fe}_{1-x} \mathrm{Co}_{x} \mathrm{Si}$. Phys. Rev. B 94, 104406 (2016).

42. Bannenberg, L. J. et al. Magnetic relaxation phenomena in the chiral magnet $\mathrm{Fe}_{1}$ ${ }_{-} \mathrm{Co}_{x} \mathrm{Si}$ : an ac susceptibility study. Phys. Rev. B 94, 134433 (2016).

43. Karube, $K$. et al. Robust metastable skyrmions and their triangular-square lattice structural transition in a high-temperature chiral magnet. Nat. Mater. 15, 1237-1242 (2016).

44. Nakajima, T. et al. Skyrmion lattice structural transition in MnSi. Sci. Adv. 3 , e1602562 (2017).

45. Ackerman, P. J. \& Smalyukh, I. I. Diversity of knot solitons in liquid crystals manifested by linking of preimages in torons and hopfions. Phys. Rev. X 7, 011006 (2017).
46. Ackerman, P. J. \& Smalyukh, I. I. Static three-dimensional topological solitons in fluid chiral ferromagnets and colloids. Nat. Mater. 16, 426-432 (2016).

47. Smalyukh, I. I., Lansac, Y., Clark, N. A. \& Trivedi, R. P. Three-dimensional structure and multistable optical switching of triple-twisted particle-like excitations in anisotropic fluids. Nat. Mater. 9, 139-145 (2009).

48. Bannenberg, L. J. et al. Reorienta tions, relaxations, metastabilities, and multidomains of skyrmion lattices. Phys. Rev. B 96, 184416 (2017).

49. Bak, P. \& Jensen, M. H. Theory of helical magnetic structures and phase transitions in MnSi and FeGe. J. Phys. C 13, L881 (1980).

50. Bordács, S. et al. Equilibrium skyrmion lattice ground state in a polar easy-plane magnet. Sci. Rep. 7, 7584 (2017).

51. Nayak, A. K. et al. Magnetic antiskyrmions above toom temperature in tetragonal Heusler materials. Nature 548, 561 (2017).

52. Leonov, A. O., Bogdanov, A. N. \& Inoue, K. Toggle-switch-like crossover between two types of isolated skyrmions within the conical phase of cubic helimagnets. Phys. Rev. B 98, 1420 (2018).

53. Müller, J. et al. Magnetic skyrmions and skyrmion clusters in the helical phase of $\mathrm{Cu}_{2} \mathrm{OSeO}_{3}$. Phys. Rev. Lett. 119, 137201 (2017).

54. Leonov, A. O. et al. The properties of isolated chiral skyrmions in thin magnetic films. New J. Phys. 18, 065003 (2016).

55. Foster, D. et al. Composite skyrmion bags in two-dimensional materials. arXiv.org, arXiv:1806.02576 (2018).

56. Dewhurst, C. D. et al. The small-angle neutron scattering instrument D33 at the Institut Laue-Langevin. J. Appl. Crystallogr. 49, 1-14 (2016).

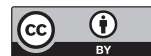

Open Access This article is licensed under a Creative Commons Attribution 4.0 International License, which permits use, sharing, adaptation, distribution and reproduction in any medium or format, as long as you give appropriate credit to the original author(s) and the source, provide a link to the Creative Commons license, and indicate if changes were made. The images or other third party material in this article are included in the article's Creative Commons license, unless indicated otherwise in a credit line to the material. If material is not included in the article's Creative Commons license and your intended use is not permitted by statutory regulation or exceeds the permitted use, you will need to obtain permission directly from the copyright holder. To view a copy of this license, visit http://creativecommons. org/licenses/by/4.0/.

(c) The Author(s) 2019 